\title{
LncRNA MEG8 plays an oncogenic role in hepatocellular carcinoma progression through miR-367-3p/14-3-3 /TGF $\beta R 1$ axis
}

\author{
Jiang LOU ${ }^{1,2}$, Wei YAN ${ }^{1,2}$, Qing-yu LI ${ }^{1,2}$, A-kao ZHU³ , Bi-qin TAN ${ }^{1,2}$, Rong DONG ${ }^{1,2}$, Xiao-zhou ZOU ${ }^{4,5, *}$, Ting LIU $U^{1,2, *}$ \\ ${ }^{1}$ Department of Pharmacy, Affiliated Hangzhou First People's Hospital, Zhejiang University School of Medicine, Hangzhou, Zhejiang, China; \\ ${ }^{2}$ Department of Clinical Pharmacy, Key Laboratory of Clinical Cancer Pharmacology and Toxicology Research of Zhejiang Province, Affili- \\ ated Hangzhou First People's Hospital, Zhejiang University School of Medicine, Hangzhou, Zhejiang, China; ${ }^{3}$ Department of General Surgery, \\ Affiliated Hangzhou First People's Hospital, Zhejiang University School of Medicine, Hangzhou, Zhejiang, China; ${ }^{4}$ Department of Pharmacy, \\ Zhejiang Provincial People's Hospital, Hangzhou, Zhejiang, China; ${ }^{5}$ People’s Hospital of Hangzhou Medical College, Hangzhou, Zhejiang, China \\ *Correspondence: zxzlovesci@163.com; 514634498@qq.com
}

Received July 30, 2020 / Accepted October 9, 2020

\begin{abstract}
Hepatocellular carcinoma (HCC) is one of the most common cancers worldwide, and it carries a poor prognosis. Clarifying the pathologic mechanisms of this disease will be beneficial for the diagnosis and treatment of HCC. LncRNA MEG8 is involved in several tumors but its role in HCC progression remains unknown. This study was designed to explore the role and regulatory mechanisms of MEG8 in HCC progression. MTT, EdU, wound-healing, and transwell assays were employed to analyze the proliferation, migration, and invasion of HCC cells. A luciferase assay was utilized to confirm the predicted binding site. RNA immunoprecipitation and co-immunoprecipitation were employed to verify the binding between MEG8

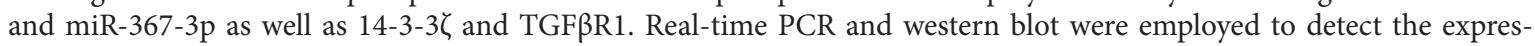
sion of interesting genes. Results revealed that MEG8 was increased in HCC tissues and cells, and was correlated with the poor prognosis of HCC patients. Inhibiting MEG8 significantly repressed the HCC cells' ability to proliferate, migrate, and

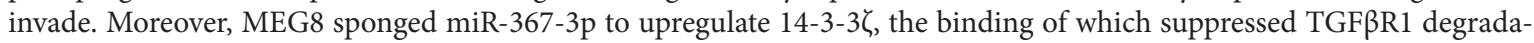
tion, thereby enhancing TGF $\beta$ signaling. In conclusion, this work exposed a novel role and regulatory mechanism of MEG8 in HCC and provided new insight into the treatment of HCC.
\end{abstract}

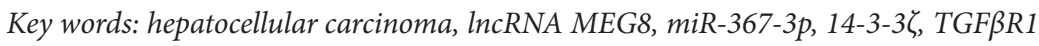

Hepatocellular carcinoma (HCC) is one of the most common cancers worldwide. Although there are a variety of therapies for HCC, such as surgical resection, radiotherapy, and chemotherapy, the prognosis remains dismal due to high recurrence rates and distant metastases [1]. Therefore, clarifying the underlying mechanisms of HCC will aid the development of new treatment strategies and improve prognoses.

Long noncoding RNAs (lncRNAs) are a class of non-coding RNAs with more than 200 nucleotides, and emerging evidence has shown that they significantly impact cancer progression [2]. Maternally expressed gene 8 (MEG8) is lncRNA located in the maternally imprinted Dlk1-Dio3 region on human chromosome $14 \mathrm{q} 32$ [3]. In recent years, a few studies have revealed the dysregulation and role of MEG8 in several tumors, such as lung cancer, pancreatic cancer, colorectal cancer, and giant cell tumors [3-5]; its role in HCC, however, remains unknown.
MicroRNAs (miRNAs) are a class of non-coding RNA molecules with $\sim 20-24$ nucleotides that exert their effects by targeting mRNA 3' UTR [6]. miR-367-3p, a member of the miR-302-367 cluster located at human chromosome 4q25 [7], is reported to be a protective factor in several cancers, such as endometrial cancer and cervical cancer $[8,9]$. In HCC, miR-367-3p is reported to enhance the sensitivity of HCC to the chemotherapeutic agent sorafenib by targeting the 3' UTR of MDM2 [10], but the role of miR-367-3p in the occurrence and development of HCC has not yet been studied.

This study focused on the role and underlying mechanism of MEG8 in HCC. The results revealed that high expression of MEG8 was correlated with a poor prognosis in HCC patients and that inhibiting MEG8 suppressed the proliferation, migration, and invasion of HCC cells. Moreover, MEG8 sponged miR-367-3p to upregulate $14-3-3 \zeta$, the binding of which suppressed TGF $\beta$ R1 degradation, thereby enhancing 
TGF $\beta$ signaling. These findings provided new insight into the treatment of HCC.

\section{Patients and methods}

Patients and tissue samples. This study was approved by the Ethics Committee of Hangzhou First People's Hospital (Hangzhou, China). Written informed consent was obtained from all patients. Seventy-four pairs of HCC tissues and adjacent normal tissues were obtained from primary HCC patients who underwent surgical resection at Hangzhou First People's Hospital between August 2010 and January 2014. None of the patients received chemotherapy, immunotherapy, or radiotherapy before the surgical resection. All samples were immediately frozen in liquid nitrogen and stored at $-80^{\circ} \mathrm{C}$. The clinicopathological features of the 74 patients are presented in Table 1.

Cell culture. Human LO2 hepatocytes and HepG2, Huh7, HCCLM3, and HMCC-97H HCC cells were purchased from the Institute of Cell Biology, Shanghai Institute for Biological

Table 1. Correlation between clinicopathological characteristics of HCC and MEG8 expression.

\begin{tabular}{|c|c|c|c|c|}
\hline \multirow[b]{2}{*}{ Characteristics } & \multirow{2}{*}{$\begin{array}{l}\text { Cases } \\
(n=74)\end{array}$} & \multicolumn{2}{|c|}{ Number of patients } & \multirow[b]{2}{*}{ p-value } \\
\hline & & $\begin{array}{c}\text { MEG8 }^{\text {high }} \\
(\mathrm{n}=37)\end{array}$ & $\begin{array}{c}\text { MEG8 }^{\text {low }} \\
(\mathrm{n}=37)\end{array}$ & \\
\hline \multicolumn{5}{|l|}{ Age (years) } \\
\hline$<60$ & 39 & 22 & 17 & \multirow{2}{*}{0.465} \\
\hline$\geq 60$ & 35 & 15 & 20 & \\
\hline \multicolumn{5}{|l|}{ Gender } \\
\hline Male & 62 & 32 & 30 & \multirow{2}{*}{0.676} \\
\hline Female & 12 & 5 & 7 & \\
\hline \multicolumn{5}{|l|}{$\mathrm{HBV}$} \\
\hline Absent & 16 & 6 & 10 & \multirow{2}{*}{0.317} \\
\hline Present & 58 & 31 & 27 & \\
\hline \multicolumn{5}{|c|}{ Serum AFP level (ng/ml) } \\
\hline$<200$ & 38 & 20 & 18 & \multirow{2}{*}{0.796} \\
\hline$\geq 200$ & 36 & 17 & 19 & \\
\hline \multicolumn{5}{|l|}{ Tumor size $(\mathrm{cm})$} \\
\hline$<5$ & 28 & 12 & 16 & \multirow{2}{*}{0.301} \\
\hline$\geq 5$ & 46 & 25 & 21 & \\
\hline \multicolumn{5}{|c|}{ Number of tumor nodules } \\
\hline 1 & 54 & 22 & 32 & \multirow{2}{*}{$0.016^{*}$} \\
\hline$\geq 2$ & 20 & 15 & 5 & \\
\hline \multicolumn{5}{|c|}{ Venous infiltration } \\
\hline Absent & 43 & 13 & 30 & \multirow{2}{*}{$0.009^{*}$} \\
\hline Present & 31 & 24 & 7 & \\
\hline \multicolumn{5}{|c|}{ Edmondson-Steiner grading } \\
\hline $\mathrm{I}+\mathrm{II}$ & 41 & 15 & 26 & \multirow{2}{*}{$0.031^{*}$} \\
\hline III + IV & 33 & 22 & 11 & \\
\hline \multicolumn{5}{|l|}{ TNM stage } \\
\hline $\mathrm{I}+\mathrm{II}$ & 46 & 18 & 28 & \multirow{2}{*}{$0.024^{*}$} \\
\hline III + IV & 28 & 19 & 9 & \\
\hline
\end{tabular}

${ }^{*}$ statistically significant
Sciences, China. LO2, Huh-7, and HCCLM3 were cultured in DMEM (Gibco) with 10\% fetal bovine serum (Biological Industries), $100 \mathrm{IU} / \mathrm{ml}$ penicillin, and $100 \mu \mathrm{g} / \mathrm{ml}$ streptomycin (Gibco) at $37^{\circ} \mathrm{C}$ under 5\% CO $\mathrm{CO}_{2}$ HepG2 and $\mathrm{HMCC}-97 \mathrm{H}$ were cultured in MEM medium (Gibco) with 10\% fetal bovine serum (Biological Industries), $100 \mathrm{IU} / \mathrm{ml}$ penicillin, and $100 \mu \mathrm{g} / \mathrm{ml}$ streptomycin (Gibco) at $37^{\circ} \mathrm{C}$ under $5 \% \mathrm{CO}_{2}$.

Cell treatment. MEG8 siRNA was synthesized by GeneChem (Shanghai, China), and the miR-367-3p mimic and inhibitor, 14-3-3 $\zeta$ siRNAs, and the plasmids containing the 14-3-3 gene were synthesized by RiboBio (Guangzhou, China); MG132 was purchased from Selleck (Shanghai, China). Mimics, inhibitors, siRNAs, and plasmids were transfected into cells using a ribo $\mathrm{FECT}^{\mathrm{T}} \mathrm{CP}$ transfection kit (RiboBio) according to the manufacturer's instructions. MG132 was dissolved in DMSO and added directly into the medium. After treatment for $24 \mathrm{~h}$, the following experiments were conducted.

Transwell assay. Transwell assay was used to analyze the invasion ability of HCC cells. A Boyden chamber with an $8 \mu \mathrm{m}$ pore filter inserted in 24 -well plates was used; the surface of the filter was coated with $40 \mu \mathrm{l}$ Matrigel (1:10, diluted in DMEM/MEM) at $37^{\circ} \mathrm{C}$ overnight, and $700 \mu \mathrm{l} 10 \%$ FBS DMEM/MEM was added to each lower well. Cells in $100 \mu \mathrm{L}$ DMEM/MEM without FBS were added to each upper well. After incubation for $24 \mathrm{~h}$ at $37^{\circ} \mathrm{C}$, cells on the upper surface of the filters were removed, and cells on the lower surface of the filters were fixed with $4 \%$ paraformaldehyde and stained with crystal violet. Cells were imaged under an inverted microscope.

5-ethynyl-20-deoxyuridine (EdU) assay. An EdU assay kit (RiboBio) was used to analyze the proliferation ability of the HCC cells. According to the manufacturer's instructions, cells were seeded into 96-well plates at appropriate cell densities and incubated with $50 \mu \mathrm{M}$ EdU buffer at $37^{\circ} \mathrm{C}$ for $2 \mathrm{~h}$. Next, the cells were fixed with $4 \%$ formaldehyde for $30 \mathrm{~min}$ and permeabilized with Triton X-100 (0.1\%) for $30 \mathrm{~min}$. Finally, the EdU solution was added, and the cell nuclei were stained with Hoechst. The staining results were visualized using a fluorescence microscope.

3-(4,5-dimethylthiazol-2-yl)-2,5-diphenyltetrazolium bromide (MTT) assay. An MTT assay kit (Sigma-Aldrich) was used to analyze the viability of the HCC cells. According to the manufacturer's instructions, cells were seeded into 96-well plates and incubated with $10 \mu \mathrm{l}$ MTT solution for $4 \mathrm{~h}$ at $37^{\circ} \mathrm{C}$. Next, the formazan crystals were dissolved in $100 \mu \mathrm{l}$ DMSO. Finally, Enzyme Immunoassay Analyzer was employed to detect the absorbance at $570 \mathrm{~nm}$.

Wound-healing assay. A wound-healing assay was used to analyze the migration ability of the HCC cells. Cells were seeded into 6-well plates, a wound was scratched using a $200 \mu \mathrm{l}$ tip, and then the plates were washed three times with PBS to remove the cell fragments. Finally, the cells were cultured for $48 \mathrm{~h}$, and the width of the wound was measured and used to analyze the migration ability. 
Real-time PCR. Total RNA was extracted using TRIzol reagent (Takara); then, 500 ng RNA was subjected to a reverse transcription reaction by using the PrimeScript RT reagent Kit (Takara) according to the manufacturer's instructions. Quantitative analysis was performed by using SYBR Premix Ex Taq (Takara) in an ABI Prism 7300 system (Applied Biosystems). PCR cycling conditions included an initial incubation at $95^{\circ} \mathrm{C}$ for $30 \mathrm{~s}$, followed by 40 cycles of denaturation at $95^{\circ} \mathrm{C}$ for $5 \mathrm{~s}$, then annealing at $60^{\circ} \mathrm{C}$ for $31 \mathrm{~s}$. Data analysis was performed by the comparative Ct method using the 7300 system SDS software. GAPDH and U6 were used as endogenous controls. The primers were as shown in Table 2.

Western blot. Protein was extracted using RIPA buffer with $0.1 \%$ PMSF. The protein contents in the supernatant were measured with the BCA protein assay kit. The denatured proteins $(40 \mu \mathrm{g})$ were separated by $10 \%$ or $15 \%$ SDS-PAGE and transferred to polyvinylidene fluoride membranes. After blocking, the membranes were incubated with primary antibodies overnight at $4{ }^{\circ} \mathrm{C}$ and then incubated with the corresponding HRP-labeled secondary antibody for $1 \mathrm{~h}$. The chemiluminescence signals were detected with the BeyoECL Star (Beyotime). The densitometric analysis was conducted with the ChemiDoc XRS+ system (Bio-Rad Co. Ltd. USA). GAPDH was used as a loading control. The primary

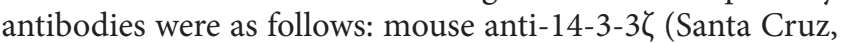
sc-293415, 1:500), mouse anti-E-cadherin (Abcam, ab1416, 1:1000), rabbit anti-Vimentin (Abcam, ab92547, 1:1000), goat anti-Snail (Abcam, ab53519, 1:500), rabbit anti-Twist1 (Abcam, ab50581, 1:1000), rabbit anti-TGFßR1 (Abcam, ab31013, 1:1000), rabbit anti-TGF $\beta$ R2 (Abcam, ab186838, 1:1000), rabbit anti-p-Smad2/3 (Abcam, ab63399, 1:500) and mouse anti-GAPDH (Beyotime, AF0006, 1:1000).

Co-immunoprecipitation. After protein extraction, $4 \mu \mathrm{g}$ of anti-14-3-3 $\zeta$ antibody was added to $1 \mathrm{ml}$ whole cell lysate containing $1000 \mu \mathrm{g}$ total protein and mixed well for pulling down the complexes bound with 14-3-3 . After overnight incubation at $4^{\circ} \mathrm{C}$, the cell lysate was further incubated with magnetic beads for $4 \mathrm{~h}$ at $4^{\circ} \mathrm{C}$ to collect the complexes. Then, western blot was conducted to detect the expression

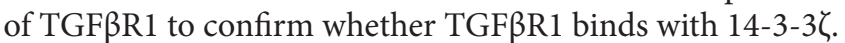

RNA immunoprecipitation (RIP). A Magna RIP kit (Millipore) was used to analyze the binding between MEG8 and miR-367-3p. Cells were lysed with RIP buffer and then incubated with anti-Ago2 antibody or IgG at $4{ }^{\circ} \mathrm{C}$ overnight. Next, the cell lysate was incubated with magnetic beads for $4 \mathrm{~h}$ at $4{ }^{\circ} \mathrm{C}$ to pull down the complexes. Finally, TRIzol reagent (Takara) was used to extract RNAs, and real-time PCR was used to detect the enrichment.

Luciferase assay. The MEG8 cDNA containing the wild-type (WT) or mutant-type (Mut) binding sequence of miR-367-3p was cloned into the luciferase reporter gene psiCHECK2. Similarly, vectors containing 14-3-3 3 3' UTR with the wild-type (WT) or mutant-type (Mut) binding sequence of miR-367-3p were also synthesized. WT-MEG8
Table 2. Primers used in the study.

\begin{tabular}{ll}
\hline MEG8 & forward: 5'CTCTGTGAATCAGGAGAGAAGA-3' \\
& reverse: 5'-TTCACCTTGGGGAAATGACC-3' \\
TGFßR1 & forward: 5'-GAGGAAAGTGGCGGGGAG-3' \\
& reverse: 5'-CCAACCAGAGCTGAGTCCAAGTA-3' \\
TGFßR2 & forward: 5'-TTTGGATGGTGGAAGGTCTC-3' \\
& reverse: 5'-GCAACAGCTATTGGGATGGT-3' \\
$\mathbf{1 4 - 3 - 3 \zeta}$ & forward: 5'-CCTGCATGAAGTCTGTAACTGAG-3' \\
& reverse: 5'-GACCTACGGGCTCCTACAACA-3' \\
GAPDH & forward: 5'-ACCCAGAAGACTGTGGATGG-3' \\
& reverse: 5'-TTCAGCTCAGGGATGACCTT-3' \\
U6 & forward: 5'-CGAGCACAGAATCGCTTCA-3' \\
& reverse: 5'-CTCGCTTCGGCAGCACATAT-3' \\
\hline
\end{tabular}

or Mut-MEG8 was co-transfected with miR-367-3p mimic or a negative control into HCCLM3 using a ribo FECT ${ }^{\text {tw }}$ CP transfection kit (RiboBio) according to the manufac-

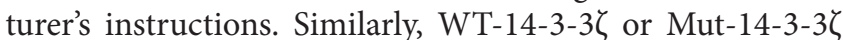
was co-transfected with miR-367-3p mimic or a negative control into HCCLM3. After transfection for $48 \mathrm{~h}$, the firefly luciferase activity was detected using the Dual-Luciferase Reporter Assay System (Promega) according to the manufacturer's protocol. The Renilla luciferase activity was used as an internal control.

Statistical analysis. SPSS 22.0 or GraphPad Prism 8.0 was used for the statistical analyses. Data were expressed as the mean \pm SD. ANOVA or a two-tailed Student's t-test was used to compare quantitative data, and nonparametric $\chi^{2}$ tests were used to compare qualitative data. The Kaplan-Meier method and log-rank tests were employed to analyze the overall or disease-free survival, and statistical significance was defined as $\mathrm{p}<0.05$.

\section{Results}

MEG8 was increased in HCC and was correlated with a poor prognosis in HCC patients. The expression of MEG8 in HCC tissues was examined first. As shown in Figure 1A, MEG8 was significantly increased compared with adjacent normal tissues. Moreover, compared with human hepatocytes (LO2), MEG8 was also increased in four types of HCC cells, with the highest expression in HCCLM3 (Figure 1B). The clinical analysis revealed that the high expression of MEG8 was strongly correlated with partial clinicopathological parameters of HCC, including Edmondson Steiner grading, venous infiltration, and the number of tumor nodules (Table 1). In addition, the correlations between MEG8 level and overall survival (OS) and disease-free survival (DFS) were analyzed. A significant negative correlation between the MEG8 level and OS or DFS was revealed (Figures 1C, 1D). All of the evidence indicated that the increase in MEG8 was closely correlated with HCC progression and a poor prognosis. 
A

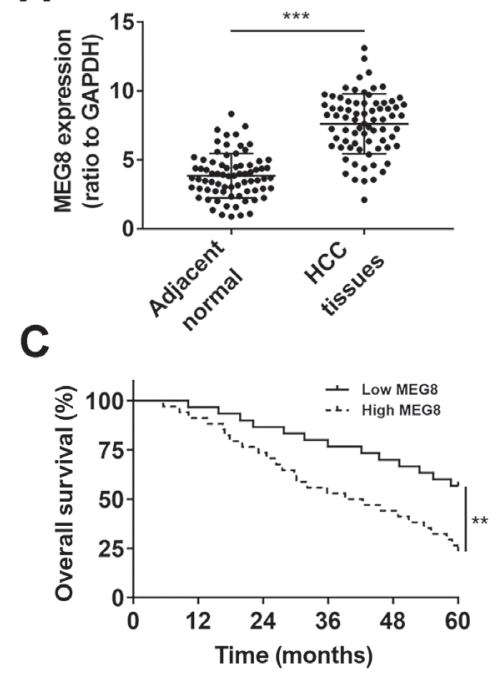

B

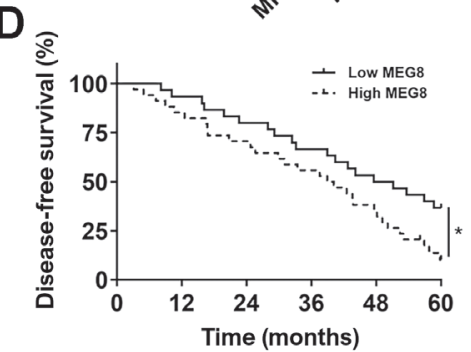

Figure 1. High expression of MEG8 was correlated with the poor prognosis of HCC patients. A) The expression of MEG8 in HCC and adjacent normal tissues detected by real-time PCR $(n=74)$. B) The expression of MEG8 in hepatocytes and HCC cells detected by real-time PCR ( $n=3$ ). C) The overall survival rate of HCC patients with high and low expression of MEG8. D) The disease-free survival rate of HCC patients with high and low expression of MEG8. ${ }^{*} \mathrm{p}<0.05,{ }^{* *} \mathrm{p}<0.01,{ }^{* * *} \mathrm{p}<0.001$

Inhibition of MEG8 suppressed proliferation, migration, and invasion of HCC cells. To investigate the role of MEG8 in HCC, MEG8 siRNA was used to inhibit the expression of MEG8 (Figure 2A). After inhibiting MEG8, the cell viability of HCCLM3 was significantly suppressed (Figure 2B). Furthermore, the EdU analysis revealed that inhibiting MEG8 repressed HCCLM3 proliferation (Figure 2C). To explore whether MEG8 mediated the migration and invasion in HCC cells, wound-healing and transwell assays were performed. As shown in Figures 2D and 2E, inhibiting MEG8 also repressed the migration and invasion in HCCLM3. These results indicated that MEG8 acted as a critical inducer in the proliferation, migration, and invasion of HCC cells.

Considering that epithelial-mesenchymal transition (EMT) is a significant contributor to migration and invasion in cancers, we next examined the role of MEG8 in EMT. As shown in Figure 2F, inhibiting MEG8 blocked EMT, as shown by the increase in E-cadherin and the decreases in Vimentin, Twist1, and Snail.

MEG8 modulated proliferation and EMT of HCC cells by sponging miR-367-3p. Bioinformatics analysis suggested that miR-367-3p was a target of MEG8 (Figure 3A). We found that miR-367-3p was significantly decreased in both HCC tissues and cells (Figures 3B, 3C). Further analysis demonstrated a prominent inverse correlation between MEG8 and miR-367-3p in HCC tissues (Figure 3D). Moreover, inhibiting MEG8 significantly increased miR-367-3p (Figure 3E). These results suggested that miR-367-3p might be a target of MEG8. To confirm this hypothesis, a luciferase assay was performed. As shown in Figures 3F and 3G, the miR-367-3p mimic significantly increased the expression of miR-367-3p and decreased the luciferase activity of wt-MEG8, but it had no effect on mut-MEG8. Considering that lncRNAs sponge miRNAs via Ago2 protein, a RIP assay was conducted. As shown in Figure 3H, both MEG8 and miR-367-3p were abundant in the pellets pulled down by the Ago2-antibody.

Next, we examined whether miR-367-3p mediated the role of MEG8 in proliferation and EMT. As shown in Figures $3 I-3 K$, the miR-367-3p inhibitor significantly repressed the expression of miR-367-3p and canceled the inhibitory effect of MEG8 siRNA on EMT and proliferation. Additionally, repressing miR-367-3p alone promoted EMT and proliferation. These results indicated that MEG8 promoted the proliferation and EMT of HCC cells by sponging miR-367-3p.

14-3-3 $\zeta$ was the target of miR-367-3p and enhanced TGF $\beta$ signaling by suppressing TGF $\beta$ R 1 degradation. $14-3-3 \zeta$, a subtype of the 14-3-3 proteins family, is critical in cancer progression [11]. Bioinformatics analysis revealed that 14-3-3 $\zeta$ might be a target of miR-367-3p (Figure 4A). We found that $14-3-3 \zeta$ was elevated in HCC tissues as well as HCC cells (Figures 4B-4D). Overexpressing miR-367-3p significantly decreased $14-3-3 \zeta$ in both mRNA and protein levels (Figures $4 \mathrm{E}, 4 \mathrm{~F}$ ). Moreover, the luciferase assay further confirmed the predicted binding site between miR-367-3p and $14-3-3 \zeta$ (Figure 4G).

Since the transforming growth factor $\beta$ (TGF $\beta$ ) signal is recognized as the key inducer of the EMT process as well as in the production of mitogenic growth factors $[12,13]$, we explored whether $14-3-3 \zeta$ mediated the TGF $\beta$ signal. After repressing $14-3-3 \zeta$ (Figure $4 \mathrm{H}$ ), the TGF $\beta$ signal was significantly reduced, characterized by decreases in TGF $\beta$ R 1 and p-Smad2/3 (Figure 4I). However, TGF $\beta$ R1 mRNA, TGF $\beta$ R2 

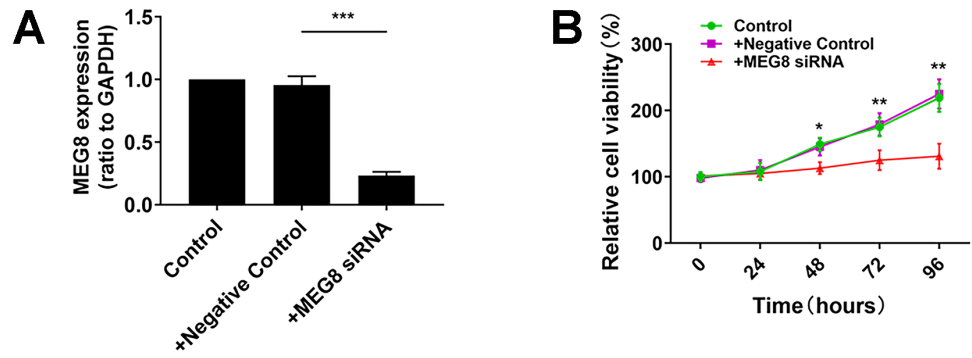
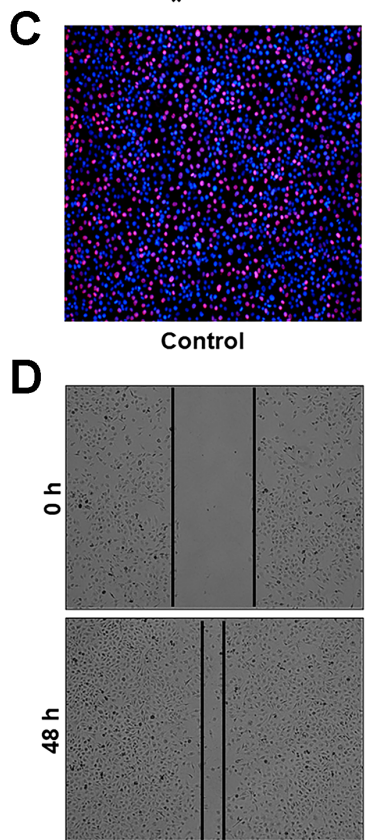

E

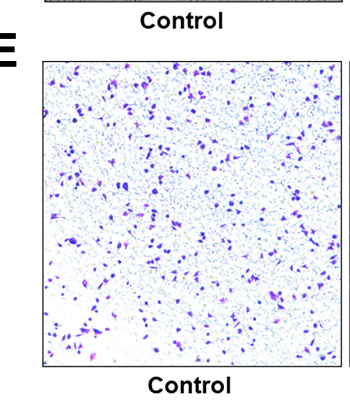

$\mathbf{F}$

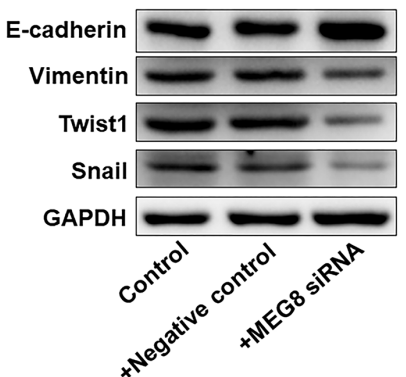

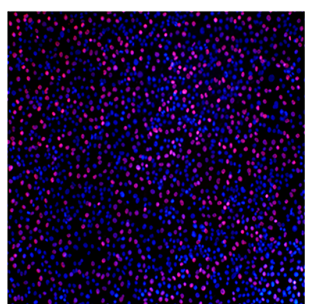

+Negative Control
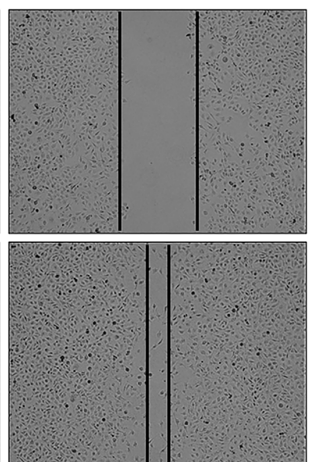

+Negative Control

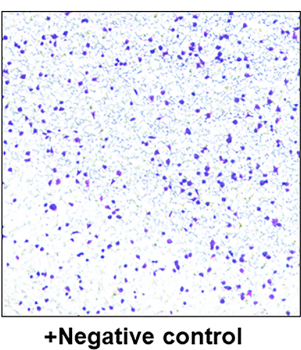

+Negative control

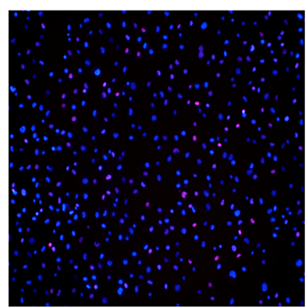

+MEG8 siRNA
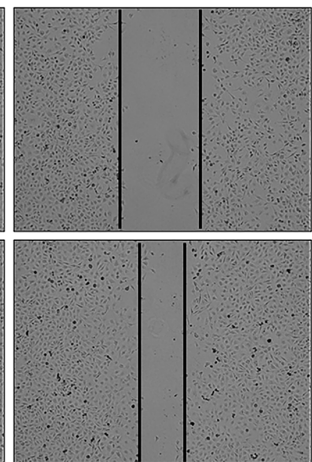

+MEG8 SiRNA
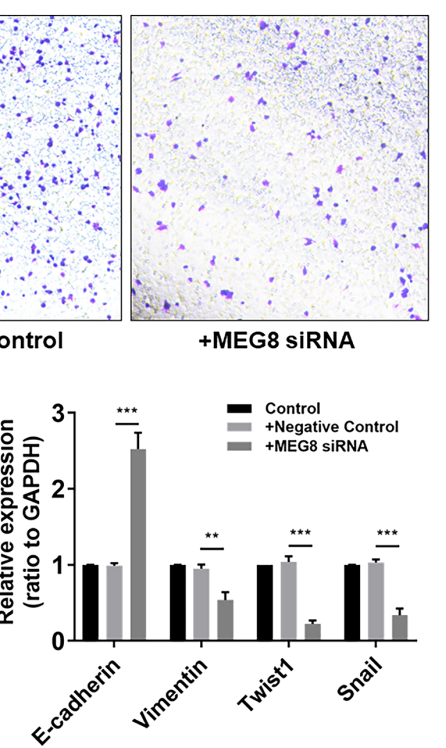
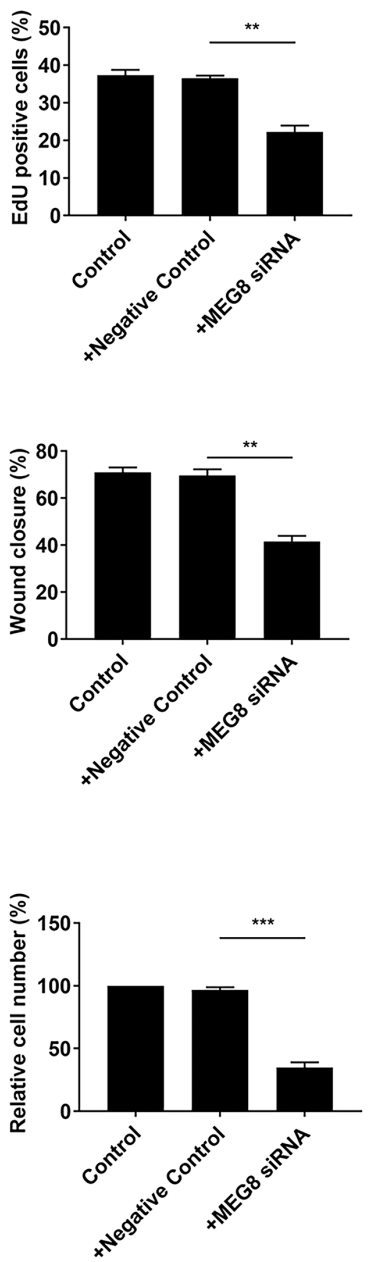

Figure 2. Inhibiting MEG8 suppressed proliferation, migration, and invasion in HCC cells. A) The efficiency of MEG8 siRNA detected by real-time PCR ( $n=3)$. B) The viability of HCCLM3 after inhibiting MEG8 detected by MTT (n=3). C) The proliferation ability of HCCLM3 after inhibiting MEG8 detected by EdU (n=3). D) The migration ability of HCCLM3 after inhibiting MEG8 detected by the wound-healing assay ( $n=3)$. E) The invasion ability of HCCLM3 after inhibiting MEG8 detected by the transwell assay $(n=3)$. F) The expression of EMT markers in HCCLM3 after inhibiting MEG8 detected by western blot $(\mathrm{n}=3) .{ }^{*} \mathrm{p}<0.05,{ }^{* *} \mathrm{p}<0.01,{ }^{* * *} \mathrm{p}<0.001$ 
A

D

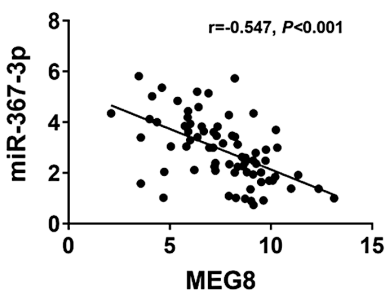

G

J

$$
\text { J }
$$

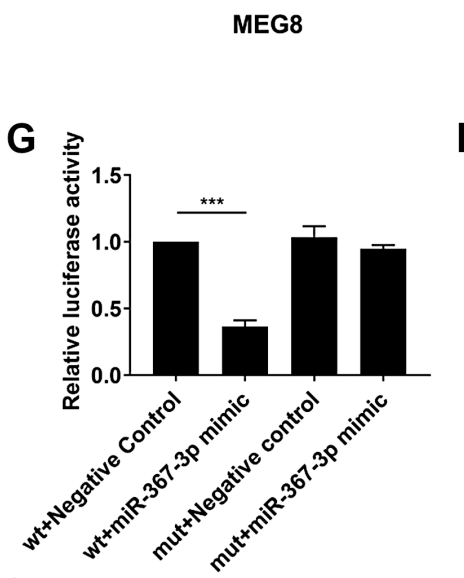

B

MEG8-wt 5'...AUGCCAUUGCCUGCAAUACAG...

miR-367-3p 3' AGUGGUAACGAUUUCACGUUAA

MEG8-mut $\quad 5^{\prime}$...AUGGTCAAUGCUGCAAUACAG...
E

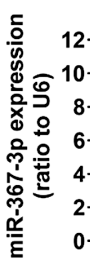

H

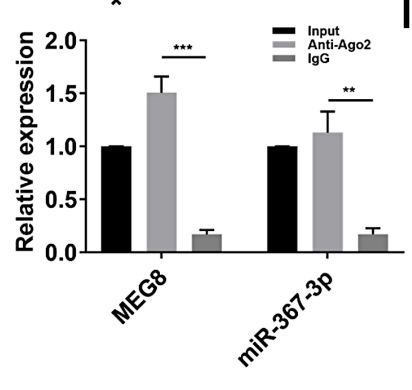

C

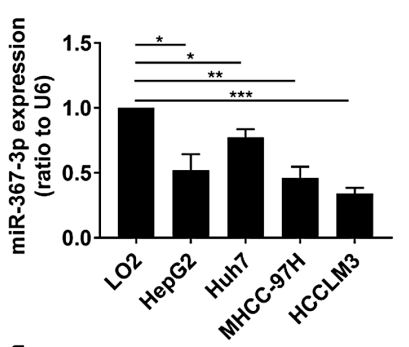

F
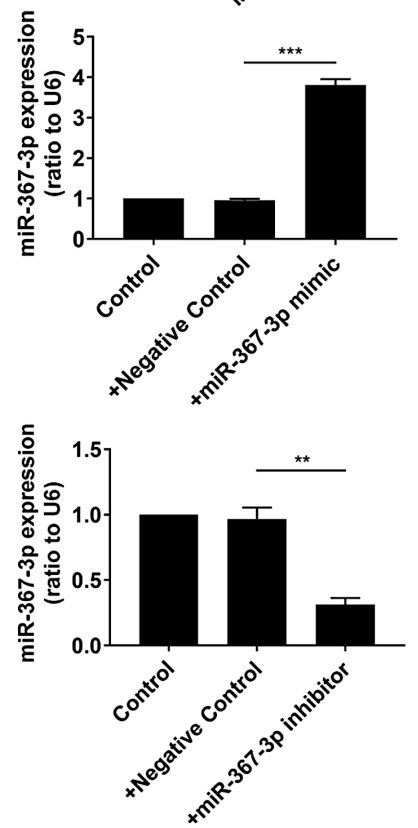
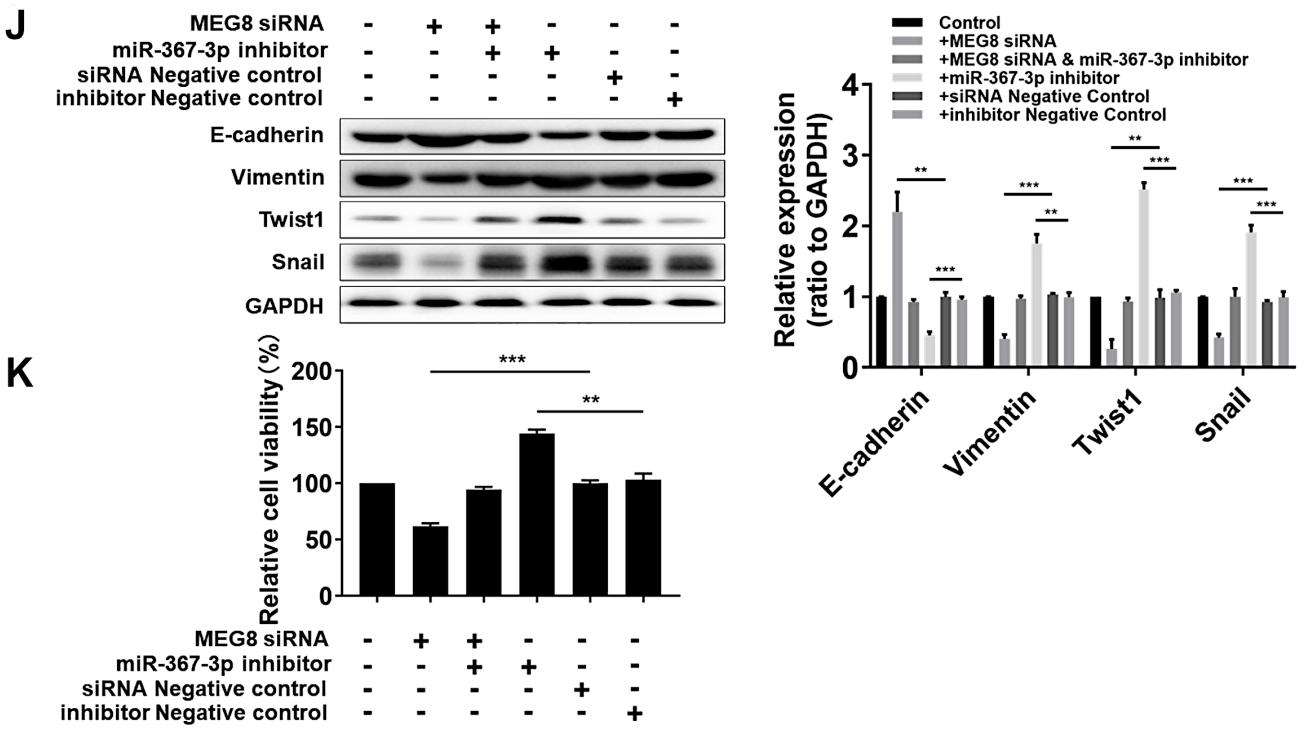

Figure 3. MEG8 mediated proliferation and EMT in HCC cells by sponging miR-367-3p. A) The predicted binding site of miR-367-3p on MEG8. B) The expression of miR-367-3p in HCC and adjacent normal tissues detected by real-time PCR ( $n=74)$. C) The expression of miR-367-3p in hepatocytes and HCC cells detected by real-time PCR $(n=3)$. D) The correlation analysis between MEG8 and miR-367-3p (n=74). E) The expression of miR-367$3 p$ in HCCLM3 after inhibiting MEG8 detected by real-time PCR (n=3). F) The efficiency of miR-367-3p mimic detected by real-time PCR (n=3). G) Luciferase analysis for examining the predicated binding site of miR-367-3p on MEG8 (n=3). H) RIP assay for miR-367-3p and MEG8 (n=3). I) The efficiency of miR-367-3p inhibitor detected by real-time PCR ( $n=3)$. J) The expression of EMT markers in HCCLM3 after inhibiting MEG8 and/or miR-367-3p detected by western blot $(n=3)$. K) The viability of HCCLM3 after inhibiting MEG8 and/or miR-367-3p detected by MTT ( $=3$ ). ${ }^{\star}$ p $<0.05$, ${ }^{* *} \mathrm{p}<0.01,{ }^{* * *} \mathrm{p}<0.001$ 


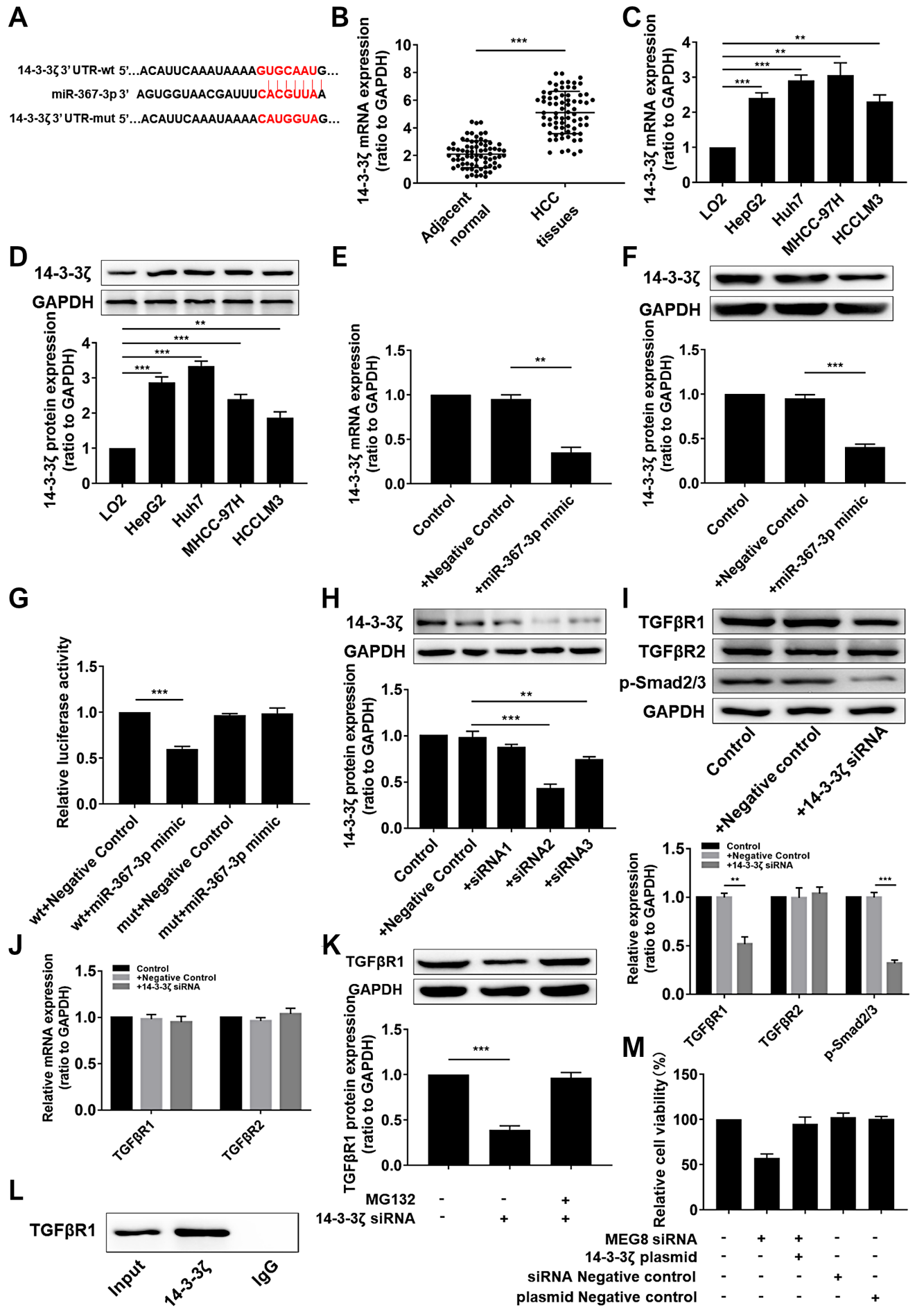

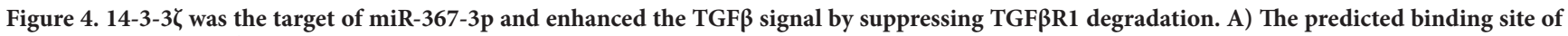
miR-367-3p on 14-3-3 $\zeta$ mRNA 3' UTR. B) The mRNA expression of 14-3-3 $\zeta$ in HCC and adjacent normal tissues detected by real-time PCR (n=74). C)

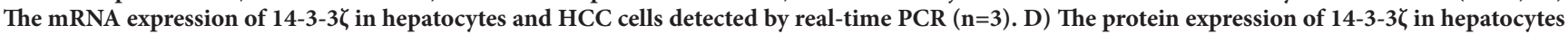
and HCC cells detected by western blot $(n=3)$. E) The mRNA expression of 14-3-3 $\zeta$ after overexpressing miR-367-3p detected by real-time PCR (n=3). F) The protein expression of 14-3-3 $\zeta$ after overexpressing miR-367-3p detected by real-time PCR $(n=3)$. G) Luciferase analysis examining the predicted

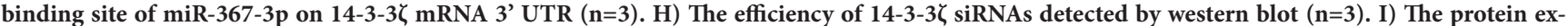

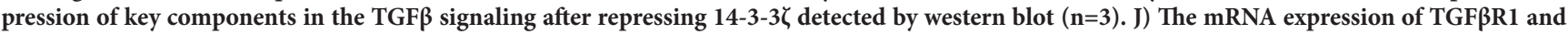

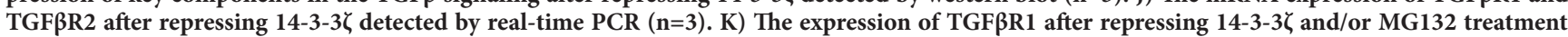
detected by western blot $(n=3)$. L) Co-immunoprecipitation analysis for examining the binding between $14-3-3 \zeta$ and $T G F \beta R 1$. ${ }^{\star *} p<0.01,{ }^{* *} p<0.001$ 
protein, and mRNA were not influenced (Figures 4I, 4J), suggesting that $14-3-3 \zeta$ might mediate the TGF $\beta$ signal by regulating TGF $\beta$ R1 protein at the posttranslational level. Previous studies have confirmed that the TGF $\beta$ R 1 protein level is mainly regulated by its internalization and ubiquitination-mediated degradation [14]. In breast cancer cells, the binding of $14-3-3 \zeta$ protects TGF $\beta$ R1 from ubiquitinationmediated degradation [15]. In this study, we found that the degradation of TGF $\beta$ R1 induced by repressing 14-3-3 $\zeta$ was canceled by the proteasome inhibitor MG132 (Figure 4K) and that $14-3-3 \zeta$ bound to TGF $\beta R 1$ (Figure $4 \mathrm{~L}$ ). Moreover, overexpressing 14-3-3 $\zeta$ canceled the inhibitory effect of MEG8 siRNA on proliferation (Figure 4M). These findings suggested that $14-3-3 \zeta$ enhanced the TGF $\beta$ signal by binding to TGF $\beta$ R1 and repressing its degradation in HCC cells.

\section{Discussion}

Increasing studies have reported that lncRNAs play a critical role in regulating cancer progression and may be a potential biomarker in cancer diagnosis and prognosis [16]. Some lncRNAs have been used for the diagnosis and prognosis of certain cancers, and some lncRNA-based strategies are being developed for anti-cancer uses. For example, the urinary PCA3 has been used for prostate cancer diagnosis with a sensitivity of $58-82 \%$ and a specificity of $56-76 \%$ [17-19]. Intravesical injection of BC-819 plasmid, which carries the gene for diphtheria toxin-A under the control of the $\mathrm{H} 19$ regulatory sequence, effectively prevents new tumor growth and prolongs recurrence time in bladder cancer patients [20].
MEG8 locates in the maternally imprinted Dlk1-Dio3 region on human chromosome 14q32 [3]. A few studies have revealed that the dysregulation of MEG8 is closely correlated with the progression of certain cancers. For instance, MEG8 is downregulated in colorectal cancer tissues and stromal cells of giant cell tumors isolated from tissue samples $[3,5]$. In contrast, in TGF $\beta$-treated lung cancer and pancreatic cancer cells, MEG8 has been shown to be increased and to act as a critical promoter in the EMT process [4]. However, no studies of MEG8 in liver cancer have been conducted until now. In this study, we found that MEG8 was increased in HCC and correlated with a poor prognosis of HCC patients, and inhibiting MEG8 suppressed the proliferation, migration, and invasion of HCC cells. However, in activated hepatic stellate cells (HSCs), injured hepatocytes (HCs), and fibrotic livers, MEG8 was found to be increased and to exert a suppressive role on the activation of HSCs and the EMT process of HCs [21], indicating that the role of MEG8 in liver diseases is complicated and worthy of further research. Mechanically, as we know, lncRNAs act as competing endogenous RNAs to sequester miRNAs. We found that miR-367-3p was a target of MEG8 and acted as a suppressor in the proliferation and EMT process in HCC cells. Similarly, in a previous study, miR-367-3p was shown to have a lower expression level in metastatic HCC patients and highly invasive HCC cells, and overexpressing miR-367-3p significantly reduced the intrahepatic and lung metastases in a mouse model with orthotopic HCC xenografts [10]. These findings exposed the key role of miR-367-3p in HCC progression.

The 14-3-3 protein family is a class of acidic soluble proteins $(28-33 \mathrm{kDa})$ that includes seven isoforms in mammals $(\beta, \varepsilon, \eta$,

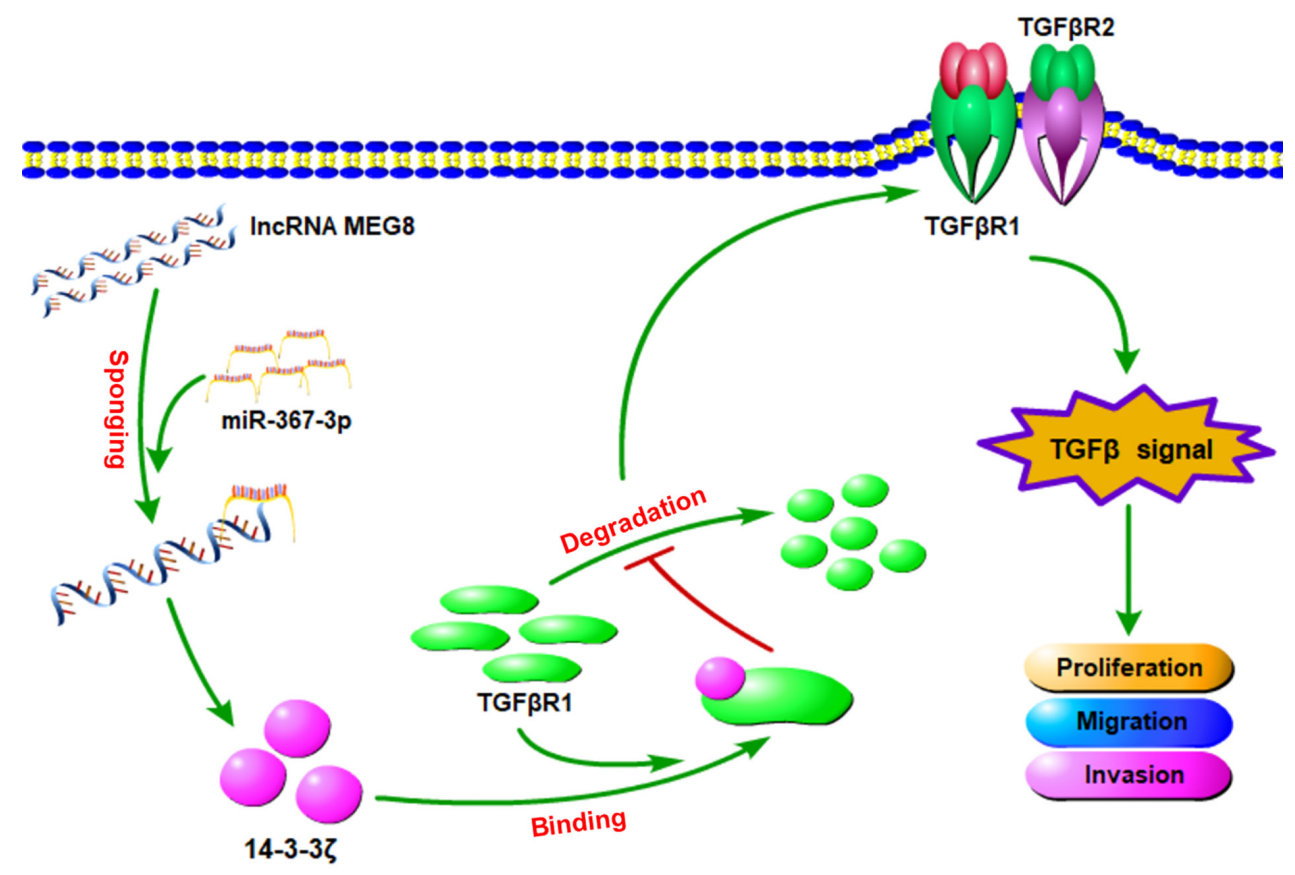

Figure 5. The regulatory mechanism of MEG8 in HCC cells. 
$\gamma, \sigma, \tau$ and $\zeta)$ [22]. The binding of 14-3-3 proteins mediates the activity, cellular localization, and stability of their targets [23]. Multiple studies have demonstrated the critical role of 14-3-3 proteins in various cancers. While most of them present tumor-promoting activity, 14-3-3 $\sigma$ exerts the opposite effect $[24,25]$. In HCC, $14-3-3 \zeta$ has been shown to be upregulated and to promote HCC progression [26, 27], but the underlying mechanism is still unclear. It is known that the TGF $\beta$ R 1 protein level is regulated mainly via its internalization and ubiquitination-mediated degradation [14]. In breast cancer, 14-3-3 $\zeta$ binds to TGF $\beta R 1$ and inhibits proteasome-mediated TGF $\beta R 1$ degradation [15]. In this study, we also found that $14-3-3 \zeta$ bound to TGF $\beta \mathrm{R} 1$ and inhibited its degradation, suggesting that $14-3-3 \zeta$ was a key enhancer of the TGF $\beta$

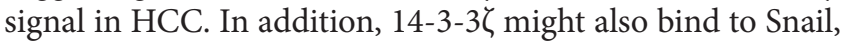
the key transcription factor of EMT, to directly mediate EMT processes in HCC, as previous studies have confirmed that most members of the 14-3-3 family, including $\zeta$, bind to Snail in 293 cells [28]. In addition, the other isoforms of 14-3-3 proteins were also confirmed to be critical inducers of proliferation, migration, and invasion in HCC [29], suggesting that 14-3-3 proteins might be key therapeutic targets in HCC. Recently, two novel compounds, RB-011 and RB-012, were found to repress lung cancer progress in vitro and in vivo by disrupting 14-3-3 dimers [30]; while their role in HCC has not yet been studied, it may be worth further examination.

This study confirmed the role of MEG8 in the proliferation, migration, and invasion of HCC cells for the first time and uncovered a novel regulatory mechanism in which MEG8 enhanced TGF $\beta$ signaling by sponging miR-367-3p and canceling 14-3-3 $\zeta$ suppression (Figure 5). This work provided new insight into the treatment of HCC.

Acknowledgments: This work was supported by the Key Medical Discipline of Zhejiang Province (2018, No. 2) and the Key Laboratory of Clinical Cancer Pharmacology and Toxicology Research of Zhejiang Province (No. 2020E10021).

\section{References}

[1] FORNER A, REIG M, BRUIX J. Hepatocellular carcinoma. Lancet 2018; 391: 1301-1314. https://doi.org/10.1016/ S0140-6736(18)30010-2

[2] KOPP F, MENDELL JT. Functional Classification and Experimental Dissection of Long Noncoding RNAs. Cell 2018; 172: 393-407. https://doi.org/10.1016/j.cell.2018.01.011

[3] LEHNER B, KUNZ P, SAEHR H, FELLENBERG J. Epigenetic silencing of genes and microRNAs within the imprinted Dlk1-Dio3 region at human chromosome 14.32 in giant cell tumor of bone. BMC Cancer 2014; 14: 495. https://doi. org/10.1186/1471-2407-14-495

[4] TERASHIMA M, ISHIMURA A, WANNA-UDOM S, SUZUKI T. MEG8 long noncoding RNA contributes to epigenetic progression of the epithelial-mesenchymal transition of lung and pancreatic cancer cells. J Biol Chem 2018; 293: 18016-18030. https://doi.org/10.1074/jbc.RA118.004006
[5] KALMÁR A, NAGY ZB, GALAMB O, CSABAI I, BODOR $A$ et al. Genome-wide expression profiling in colorectal cancer focusing on lncRNAs in the adenoma-carcinoma transition. BMC Cancer 2019; 19: 1059. https://doi.org/10.1186/ s12885-019-6180-5

[6] BARTEL DP. MicroRNAs: genomics, biogenesis, mechanism, and function. Cell 2004; 116: 281-297. https://doi. org/10.1016/s0092-8674(04)00045-5

[7] MURRAY MJ, HUDDART RA, COLEMAN N. The present and future of serum diagnostic tests for testicular germ cell tumours. Nat Rev Urol 2016; 13: 715-725. https://doi. org/10.1038/nrurol.2016.170

[8] YANG T, TIAN S, WANG L, WANG Y, ZHAO J. MicroRNA-367-3p overexpression represses the proliferation and invasion of cervical cancer cells through downregulation of SPAG5-mediated Wnt/ $\beta$-catenin signalling. Clin Exp Pharmacol Physiol 2020; 47: 687-695. https://doi. org/10.1111/1440-1681.13222

[9] MA J, LI D, KONG FF, YANG D, YANG H et al. miR-302a5p/367-3p-HMGA2 axis regulates malignant processes during endometrial cancer development. J Exp Clin Cancer Res 2018; 37: 19. https://doi.org/10.1186/s13046-018-0686-6

[10] XU J, LIN H, LI G, SUN Y, CHEN J et al. The miR-367$3 p$ Increases Sorafenib Chemotherapy Efficacy to Suppress Hepatocellular Carcinoma Metastasis through Altering the Androgen Receptor Signals. EBioMedicine 2016; 12: 55-67. https://doi.org/10.1016/j.ebiom.2016.07.013

[11] FAN X, CUI L, ZENG Y, SONG W, GAUR U et al. 14-3-3 Proteins Are on the Crossroads of Cancer, Aging, and AgeRelated Neurodegenerative Disease. Int J Mol Sci 2019; 20: 3518. https://doi.org/10.3390/ijms20143518

[12] LAMOUILLE S, XU J, DERYNCK R. Molecular mechanisms of epithelial-mesenchymal transition. Nat Rev Mol Cell Biol 2014; 15: 178-196. https://doi.org/10.1038/nrm3758

[13] FABREGAT I, FERNANDO J, MAINEZ J, SANCHO P. TGF-beta signaling in cancer treatment. Curr Pharm Des 2014; 20: 2934-2947. https://doi.org/10.2174/138161281131 99990591

[14] DI GUGLIELMO GM, LE ROY C, GOODFELLOW AF, WRANA JL. Distinct endocytic pathways regulate TGF-beta receptor signalling and turnover. Nat Cell Biol 2003; 5: 410421. https://doi.org/10.1038/ncb975

[15] LU J, GUO H, TREEKITKARNMONGKOL W, LI P, ZHANG J et al. 14-3-3 Cooperates with ErbB2 to Promote Progression of Ductal Carcinoma in Situ to Invasive Breast Cancer by Inducing Epithelial-Mesenchymal Transition. Cancer Cell 2009; 16: 195-207. https://doi.org/10.1016/j. ccr.2009.08.010

[16] GUPTA SC, TRIPATHI YN. Potential of long non-coding RNAs in cancer patients: From biomarkers to therapeutic targets. Int J Cancer 2017; 140: 1955-1967. https://doi. org/10.1002/ijc.30546

[17] MARKS LS, FRADET Y, DERAS IL, BLASE A, MATHIS $J$ et al. PCA3 molecular urine assay for prostate cancer in men undergoing repeat biopsy. Urology 2007; 69: 532-535. https://doi.org/10.1016/j.urology.2006.12.014 
[18] VAN GILS MPMQ, HESSELS D, VAN HOOIJ O, JANNINK SA, PEELEN WP et al. The time-resolved fluorescence-based PCA3 test on urinary sediments after digital rectal examination; a Dutch multicenter validation of the diagnostic performance. Clin Cancer Res 2007; 13: 939-943. https://doi.org/10.1158/1078-0432.CCR-06-2679

[19] TINZL M, MARBERGER M, HORVATH S, CHYPRE C. DD3PCA3 RNA analysis in urine--a new perspective for detecting prostate cancer. Eur Urol 2004; 46: 182-186; discussion 187. https://doi.org/10.1016/j.eururo.2004.06.004

[20] GOFRIT ON, BENJAMIN S, HALACHMI S, LEIBOVITCH I, DOTAN Z et al. DNA based therapy with diphtheria toxin-A BC-819: a phase $2 \mathrm{~b}$ marker lesion trial in patients with intermediate risk non-muscle invasive bladder cancer. J Urol 2014; 191: 1697-1702. https://doi.org/10.1016/j. juro.2013.12.011

[21] CHEN T, LIN H, CHEN X, LI G, ZHAO Y et al. LncRNA Meg8 suppresses activation of hepatic stellate cells and epithelial-mesenchymal transition of hepatocytes via the Notch pathway. Biochem Biophys Res Commun 2020; 521: 921927. https://doi.org/10.1016/j.bbrc.2019.11.015

[22] MHAWECH P. 14-3-3 proteins--an update. Cell Res 2005; 15: 228-236. https://doi.org/10.1038/sj.cr.7290291

[23] TZIVION G, AVRUCH J. 14-3-3 proteins: active cofactors in cellular regulation by serine/threonine phosphorylation. J Biol Chem 2002; 277: 3061-3064. https://doi.org/10.1074/ jbc.R100059200

[24] MORRISON DK. The 14-3-3 proteins: integrators of diverse signaling cues that impact cell fate and cancer development. Trends Cell Biol 2009; 19: 16-23. https://doi.org/10.1016/j. tcb.2008.10.003
[25] PHAN L, CHOU PC, VELAZQUEZ-TORRES G, SAMUDIO I, PARRENO $\mathrm{K}$ et al. The cell cycle regulator $14-3-3 \sigma$ opposes and reverses cancer metabolic reprogramming. Nat Commun 2015; 6: 7530. https://doi.org/10.1038/ncomms8530

[26] TANG Y, WANG R, ZHANG Y, LIN S, QIAO N et al. CoUpregulation of 14-3-3 and P-Akt is Associated with Oncogenesis and Recurrence of Hepatocellular Carcinoma. Cell Physiol Biochem 2018; 45: 1097-1107. https://doi. org/10.1159/000487351

[27] TANG Y, LIU S, LI N, GUO W, SHI J et al. 14-3-3 $\zeta$ promotes hepatocellular carcinoma venous metastasis by modulating hypoxia-inducible factor-1a. Oncotarget 2016; 7: 1585415867. https://doi.org/10.18632/oncotarget.7493

[28] HOU Z, PENG H, WHITE DE, WANG P, LIEBERMAN $\mathrm{PM}$ et al. 14-3-3 binding sites in the Snail protein are essential for Snail-mediated transcriptional repression and epithelial-mesenchymal differentiation. Cancer Res 2010; 70: 4385-4393. https://doi.org/10.1158/0008-5472.CAN-100070

[29] WU YJ, JAN YJ, KO BS, LIANG SM, LIOU JY. Involvement of 14-3-3 Proteins in Regulating Tumor Progression of Hepatocellular Carcinoma. Cancers (Basel) 2015; 7: 1022-1036. https://doi.org/10.3390/cancers7020822

[30] WOODCOCK JM, COOLEN C, GOODWIN KL, BAEK DJ, BITTMAN R et al. Destabilisation of dimeric 14-3-3 proteins as a novel approach to anti-cancer therapeutics. Oncotarget 2015; 6: 14522-14536. https://doi.org/10.18632/ oncotarget.3995 